\title{
Double-pass and interferometric measures of the optical quality of the eye
}

\author{
David R. Williams \\ Center for Visual Science, University of Rochester, Rochester, New York 14627 \\ David H. Brainard \\ Department of Psychology, University of California, Santa Barbara, Santa Barbara, California 93106 \\ Matthew J. McMahon \\ Center for Visual Science, University of Rochester, Rochester, New York 14627 \\ Rafael Navarro \\ Instituto de Optica, Consejo Superior de Investigaciones Científicas, Serrano 121, 28006 Madrid, Spain
}

Received January 3, 1994; revised manuscript received June 20, 1994; accepted July 29, 1994

\begin{abstract}
We compare two methods for measuring the modulation transfer function (MTF) of the human eye: an interferometric method similar to that of Campbell and Green [J. Physiol. (London) 181,576 (1965)] and a double-pass procedure similar to that of Santamaria et al. [J. Opt. Soc. Am. A 4, 1109 (1987)]. We implemented various improvements in both techniques to reduce error in the estimates of the MTF. We used the same observers, refractive state, pupil size $(3 \mathrm{~mm})$, and wavelength $(632.8 \mathrm{~nm})$ for both methods. In the double-pass method we found close agreement between the plane of subjective best focus for the observer and the plane of objective best focus, suggesting that much of the reflected light is confined within individual cones throughout its double pass through the receptor layer. The double-pass method produced MTF's that were similar to but slightly lower than those of the interferometric method. This additional loss in modulation transfer is probably attributable to light reflected from the choroid, because green light, which reduces the contribution of the choroid to the fundus reflection, produces somewhat higher MTF's that are consistent with the interferometric results. When either method is used, the MTF's lie well below those obtained with the aberroscope method [Vision Res. 28, 659 (1988)]. On the basis of the interferometric method, we propose a new estimate of the monochromatic MTF of the eye.
\end{abstract}

\section{INTRODUCTION}

A complete description of human spatial vision requires an accurate characterization of the optical performance of the human eye. Here we compare two techniques for assessing the eye's optical quality. The modulation transfer function (MTF) can be measured with the doublepass method, ${ }^{1-6}$ in which a point source is imaged on the retina and the light that is reflected out of the eye is imaged a second time. This aerial image is captured and used to compute the MTF for a single pass through the eye's optics. With the interferometric method the MTF is estimated from the ratio of contrast sensitivity to conventional gratings and interference fringes that are not blurred by the optics. ${ }^{7-9}$

To our knowledge, these techniques have never been compared under the same experimental conditions. Campbell and Gubisch ${ }^{4}$ pointed out that their doublepass MTF's were lower than the interferometric results of Campbell and Green. ${ }^{7}$ They attributed this difference to light scattered from ocular layers other than the photoreceptors in the double-pass method. However, as Campbell and Gubisch suggested, other factors, such as individual differences among the small numbers of observers in the two studies, could account for this difference. In this paper we compare the MTF's obtained with improved double-pass and interferometric methods, using the same observers, pupil size $(3 \mathrm{~mm})$, refractive state, wavelength, and retinal location. We show that in red light $(632.8 \mathrm{~nm})$ the double-pass technique provides lower estimates of the MTF than does the interferometric method. We argue that in red light the interferometric method provides the better estimate of the singlepass MTF of the human eye. We analyze the additional sources of image blur in the double-pass method and suggest that with this method the use of green light provides a better estimate of the MTF.

\section{INTERFEROMETRIC METHOD}

\section{A. Observers}

Measurements were made on three observers: RNB, DRW, and DHB, aged 34,38 , and 32 years, respectively. They were mildly myopic $(0.2,1.6$, and $0.4 \mathrm{D}$, respectively). In addition, DRW had $0.8 \mathrm{D}$ of astigmatism. These refractive errors were corrected during the measurements, as described below.

\section{B. Apparatus}

Figure 1 shows the optical system. Two identical interference fringes, $\mathrm{A}$ and $\mathrm{B}$, were produced with $\mathrm{a}$ 


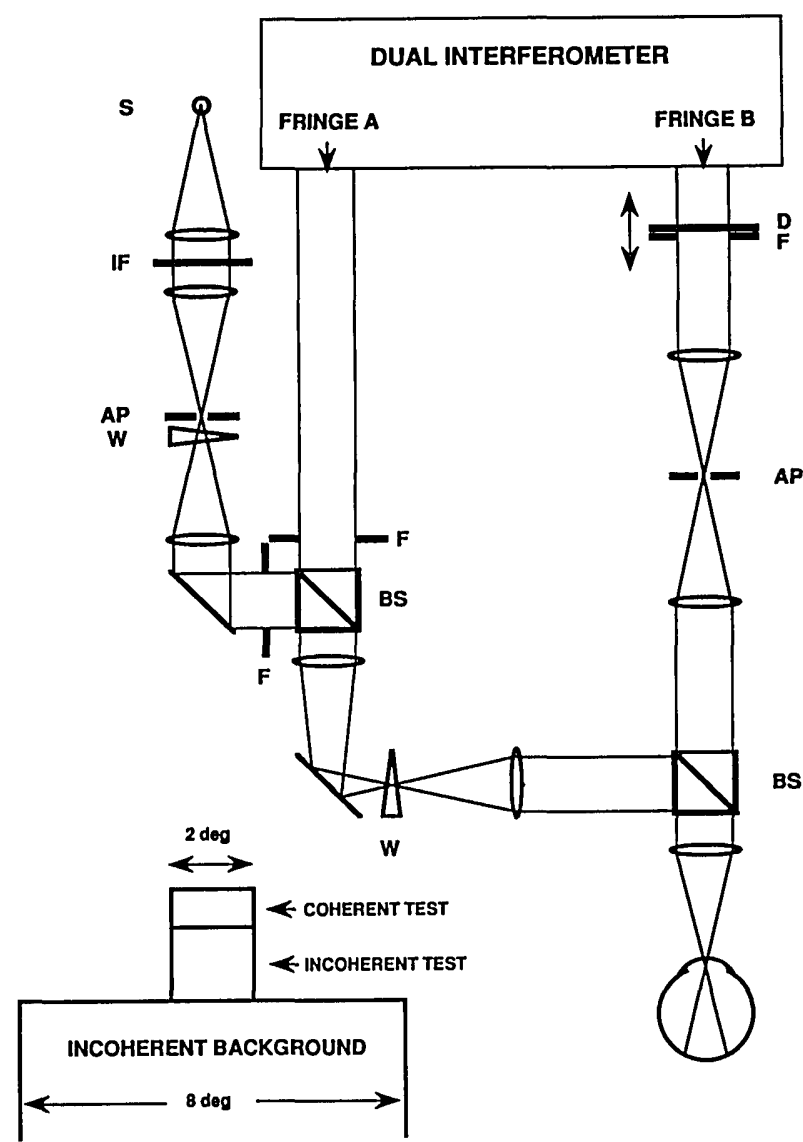

Fig. 1. Diagram of interferometric apparatus. An intensity profile of the stimulus display is shown in the inset at the bottom. The dual interferometer produces two collimated fields, each containing an interference fringe generated from a separate laser source. Fringe A is an interference fringe cast on the retina; fringe $B$ is cast on a rotating diffuser, $D$, which results in an incoherent grating cast on the retina. $S$, tungsten source; IF, 630-nm interference filter; AP, 3-mm artificial pupil, which is conjugate with the natural pupil; $\mathrm{W}$, neutral-density wedge; F's, field stops, which are conjugate with the retina; BS, beam splitters.

dual-polarization interferometer. This device, which is described by Sekiguchi et al., ${ }^{10}$ permits forced-choice contrast sensitivity measurements without blurring by the optics of the eye. Each interferometer generated a collimated beam containing the interference fringe. Each interferometer had its own $\mathrm{He}-\mathrm{Ne}$ laser source $(632.8 \mathrm{~nm})$. The two point sources associated with fringe A were imaged in the plane of the observer's pupil, forming a fringe on the retina that was used to make interferometric contrast sensitivity measurements. Fringe B was cast on a rotating diffuser, which scattered the light in a broad range of directions. This produced an incoherent grating and allowed us to measure incoherent contrast sensitivity. The ratio of incoherent to interferometric contrast sensitivity was taken as the MTF of the eye. The use of an interferometer to generate the incoherent grating had the advantage that the interferometer and the incoherent contrast sensitivity measurements could be made under very similar conditions. The two kinds of grating had the same wavelength, and both were seen in the same Maxwellian-view system.

The diffuser was conjugate with the retina and was viewed through a $3-\mathrm{mm}$ artificial pupil, which was con- jugate with the natural pupil of the eye. The diffuser was coarse enough to remove any traces of the pair of interferometric point sources in the pupil plane and to fill the 3-mm artificial pupil uniformly with light. During the contrast sensitivity measurements, the diffuser was rotated rapidly enough that its motion could not be seen in the grating field. We replaced the eye with a CCD camera and recorded images of the incoherent gratings. The contrast of these images was reduced only by diffraction at the $3-\mathrm{mm}$ artificial pupil, showing that aberrations and scatter in the apparatus were negligible.

\section{Procedure}

Accommodation was paralyzed with two drops of cyclopentolate hydrochloride (1\%). During the alignment procedure at the beginning of each session, observers adjusted the horizontal and vertical positions of the eye to optimize the image quality of a high-frequency incoherent grating. The observer then optimized the focus of the incoherent grating by sliding the rotating diffuser axially. We checked grating focus periodically throughout the experiment to correct for any drift in the refractive state of the eye. An additional drop of cyclopentolate was instilled if required. Gratings used in all the measurements were horizontal.

Unlike incoherent gratings, interference fringes contain laser speckle. This speckle can cause masking that reduces contrast sensitivity. ${ }^{11}$ We adopted two procedures to eliminate the effect of speckle on our MTF measurements. First, as shown in the inset of Fig. 1, the 2-deg test field was superimposed upon an 8-deg incoherent background of speckle-free 630-nm light. This procedure reduces speckle masking. ${ }^{11}$ Second, the test field consisted of the sum of the fields that produced the interferometric and the incoherent gratings. Both of these fields, as well as the background, were present throughout the measurements. The interferometric contrast sensitivity function was obtained by modulation of the coherent field while the contrast of the incoherent field was kept at zero. The incoherent CSF was obtained by modulation of the incoherent field while the contrast of the coherent field was kept at zero. This procedure ensured that any residual spatial noise would have produced the same masking effect on both the coherent and the incoherent measurements. Since the MTF depends on the ratio of the measurements, any residual speckle masking would not affect the MTF estimate.

For a given spatial frequency the relative intensities of the three superimposed fields was set as follows. The contrast of both superimposed gratings was set to $100 \%$. The intensity of the interference fringe was adjusted with a neutral-density wedge until the gratings had equal subjective contrast. The incoherent background was then increased in intensity until the two gratings were just above threshold. The total retinal illuminance of the stimulus varied from spatial frequency to spatial frequency but was always the same for the interferometric and the incoherent measurements at a given spatial frequency. The retinal illuminance was always greater than 900 and less than 15,000 Td.

Interferometric and incoherent contrast sensitivities were measured with forced-choice trials randomly interleaved in each run. The observer could not distinguish 
between the two types of trial. Each trial consisted of two 500-ms intervals, and the observer's task was to select the interval containing the grating or fringe. Feedback was provided. The grating contrast for each trial was determined with the QUEST procedure. ${ }^{12}$ A single spatial frequency was tested in each run, which consisted of 50 trials of each of the two stimuli. Measurements of the three field irradiances were made after each run for the purpose of computing threshold contrast. Typically, all spatial frequencies for a given retinal location were tested in a day-long session. Three sessions were completed by each of the three observers. As a check on our method, we measured the MTF of one observer (DRW) with a 1-mm artificial pupil, for which the human eye is diffraction limited. Within experimental error our results agreed with the diffraction prediction.

\section{INTERFEROMETRIC RESULTS}

The symbols in Fig. 2 show the mean MTF for each of the three observers. The means and the standard errors of the means are shown in Table 1 . The solid curve in Fig. 2 is a least-squares fit of the product of

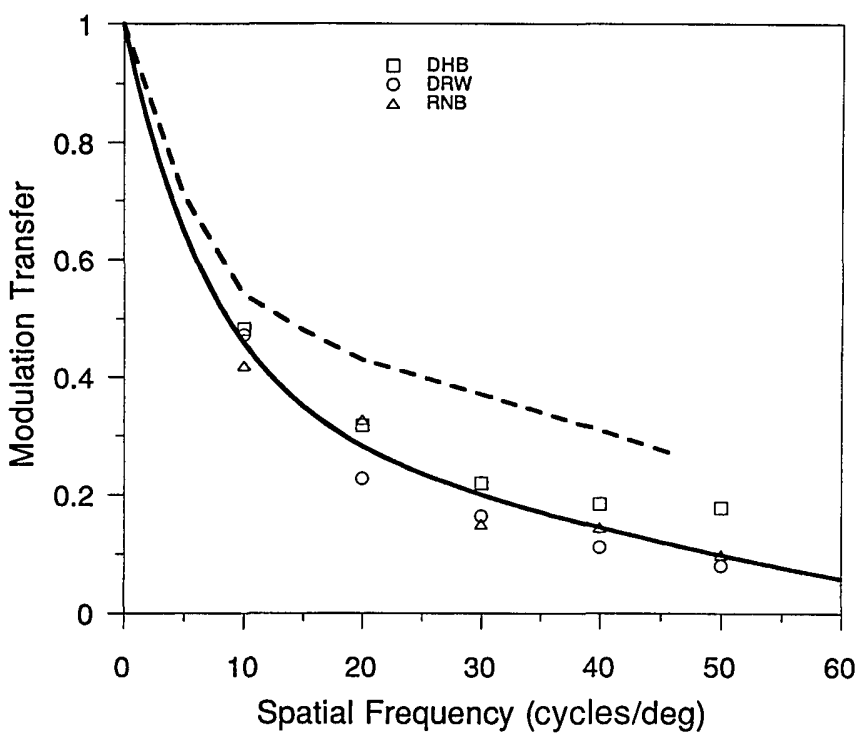

Fig. 2. Interferometric MTF's for three observers, with 3-mm pupil and 632.8-nm wavelength. Solid curve, least-squares fit of the product of an exponential and the diffraction-limited MTF; dashed curve, interferometric MTF of Campbell and Green, ${ }^{7}$ obtained with a $2.8-\mathrm{mm}$ pupil. the diffraction-limited MTF for a 3-mm pupil in 632.8$\mathrm{nm}$ light and the sum of a constant and an exponential. The function fitted to the data has the form

$$
M\left(s, s_{0}\right)=D\left(s, s_{0}\right)\left[w_{1}+w_{2} \exp (-a s)\right]
$$

where $M\left(s, s_{0}\right)$ is the modulation transfer, $s$ is spatial frequency in cycles per degree, and $s_{0}$ is the incoherent cutoff frequency for a diffraction-limited imaging system with a circular pupil (82.7 cycles/deg at $632.8 \mathrm{~nm}$ with a 3-mm pupil). The modulation transfer, $D\left(s, s_{0}\right)$, for such a diffraction-limited system is ${ }^{13}$

$$
\begin{array}{r}
D\left(s, s_{0}\right)=\frac{2}{\pi}\left[\cos ^{-1}\left(\frac{s}{s_{0}}\right)-\left(\frac{s}{s_{0}}\right) \sqrt{1-\left(\frac{s}{s_{0}}\right)^{2}}\right] \\
\text { for } s<s_{0}
\end{array}
$$

We chose Eq. (1) to represent our data because the component corresponding to diffraction captures the fact that the MTF must fall to zero at the diffraction limit. The term corresponding to a constant plus an exponential is required for dropping the curve below the diffraction limit. We do not attach any theoretical significance to this particular term. The parameters yielding the least-squares fit were $a=0.1212, w_{1}=0.3481$, and $w_{2}=0.6519$.

The dashed curve in Fig. 2 shows the MTF for a single observer obtained by Campbell and Green ${ }^{7}$ with a $2.8-\mathrm{mm}$ pupil. The ratios of incoherent to interferometric contrast sensitivity in our study are consistently lower than the data of Campbell and Green. One possible reason is individual differences. It may also have been that the interferometric field used by Campbell and Green contained masking noise that was absent from the CRT that they used to display incoherent gratings. This might have made the interferometric and the incoherent contrast sensitivities more similar, which would have increased the modulation transfer computed from their ratio. Our use of a single stimulus field for both coherent and incoherent contrast sensitivity measurements ensured that differences in spatial noise between the two component fields did not distort our estimates. Our method also allowed interferometric and incoherent contrast sensitivities to be measured at the same wavelength, whereas the spectral distributions for the two kinds of stimulus were different in the experiment of Campbell and Green.

\begin{tabular}{|c|c|c|c|c|c|c|c|c|}
\hline \multirow{2}{*}{$\begin{array}{c}\text { Spatial } \\
\text { Frequency }\end{array}$} & \multirow{2}{*}{$\begin{array}{c}\text { Average } \\
\text { MTF }\end{array}$} & \multirow{2}{*}{$\begin{array}{l}\text { Standard } \\
\text { Deviation }\end{array}$} & \multicolumn{2}{|c|}{ Observer DHB } & \multicolumn{2}{|c|}{ Observer DRW } & \multicolumn{2}{|c|}{ Observer RNB } \\
\hline & & & $\overline{\mathrm{MTF}}$ & SEM & MTF & $\overline{\text { SEM }}$ & $\overline{\mathrm{MTF}}$ & SEM \\
\hline 10 & 0.458 & 0.034 & 0.482 & 0.050 & 0.472 & 0.011 & 0.419 & 0.043 \\
\hline 20 & 0.291 & 0.055 & 0.317 & 0.054 & 0.228 & 0.025 & 0.327 & 0.027 \\
\hline 30 & 0.178 & 0.037 & 0.220 & 0.015 & 0.164 & 0.029 & 0.150 & 0.027 \\
\hline 40 & 0.147 & 0.037 & 0.185 & 0.013 & 0.112 & 0.018 & 0.145 & 0.021 \\
\hline 50 & 0.119 & 0.052 & 0.178 & 0.025 & 0.080 & 0.014 & 0.099 & 0.024 \\
\hline
\end{tabular}

Table 1. Tabulated Values of the Interferometric MTF Averaged across Three Observers and Standard Deviation Based on the Variability among Them ${ }^{a}$

${ }^{a}$ Individual MTF's are also tabulated for each observer, along with the standard error of the mean, SEM, based on the variability between estimates of the MTF from three experimental sessions. 


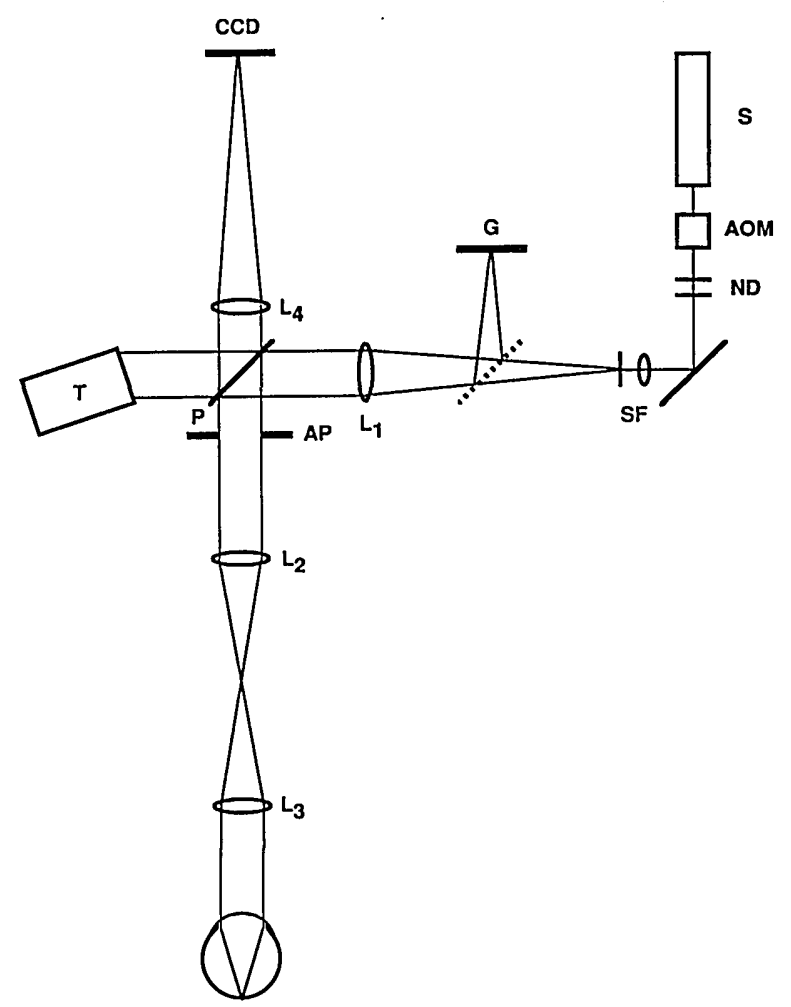

Fig. 3. Diagram of the double-pass apparatus. $\mathrm{S}, \mathrm{He}-\mathrm{Ne}$ laser source; AOM, acousto-optic modulator; ND, neutral-density filters; SF, spatial filter; G, focusing grating; AP, 3-mm artificial pupil; $P$, pellicle; $T$, light trap; $C C D$, array detector for capturing the aerial image; L's, lenses.

\section{DOUBLE-PASS METHOD}

\section{A. Apparatus}

The apparatus used in the double-pass experiments is shown in Fig. 3 . It is similar to devices developed at the Instituto de Optica in Madrid. 1,6 The main difference is that we used a cooled, single-frame CCD camera instead of a video camera to acquire aerial images of the point source on the retina. This considerably reduced the retinal irradiance required for collecting an image, because light arriving at the CCD could be integrated over many seconds. The total energy incident upon the cornea that was required for making an exposure was approximately $1.5 \times 10^{-6} \mathrm{~J}$, which was distributed over $5 \mathrm{~s}$. This irradiance is 3.6 orders of magnitude below the ANSI Z-136.1 maximum permissible exposure limit. ${ }^{14}$

The source was a $\mathrm{He}-\mathrm{Ne}$ laser $(632.8 \mathrm{~nm}, 20 \mathrm{~mW})$. The beam passed through an acousto-optic modulator AOM, that controlled the exposure duration, which was $5 \mathrm{~s}$. The AOM had an extinction ratio of $10^{-4}$, which allowed enough light through when the beam was nominally off to provide the observer with a fixation target. The beam then passed through neutral-density filters, followed by a spatial filter. The spatial filter consisted of a $10 \times$ microscope objective that focused the beam onto a $25-\mu \mathrm{m}$ pinhole. The pinhole formed the point source that was conjugate with the retina and subtended $0.34^{\prime}$ of arc. The emerging beam was collimated by lens $\mathrm{L}_{1}(f=254 \mathrm{~mm})$. A pellicle reflected $10 \%$ of the light toward the eye, with the transmitted light absorbed by a light trap. The light passed through a 3-mm artifi- cial pupil that was conjugate with the observer's natural pupil. This process controlled pupil size while avoiding the complication of placing an artificial pupil in front of the cornea, out of the pupil plane. Lens $\mathrm{L}_{2}(f=110 \mathrm{~mm})$ formed an image of the pinhole that the observer viewed through $\mathrm{L}_{3}(f=110 \mathrm{~mm})$. $\mathrm{L}_{3}$ lay one focal length from the observer's pupil plane.

The light that was reflected out of the eye formed an aerial image one focal length from $L_{2}$. Then $L_{2}$ and $L_{4}$ cast the aerial image on a CCD array after the light passed again through the artificial pupil. The CCD camera (Photometrics Series 200 system) contained a fullframe CCD array (Kodak KAF 1400 chip, 1.4 Mpixel). A mechanical shutter on the CCD camera was synchronized with the AOM that controlled the retinal light exposure. Aerial images were $512 \times 512$ pixels with 12 bits/ pixel. Each pixel was $13.6 \mu \mathrm{m}$ on a side and was produced by summing the signals from a $2 \times 2$ array of physical pixels on the CCD. The magnification of the retinal image could be adjusted by changing the focal length of lens $\mathrm{L}_{4}$. We used two magnifications, $15 \times$ and $30 \times$, corresponding to CCD fields of 1.6 and $0.8 \mathrm{deg}$ and pixel sampling rates of 321 and 642 cycles/deg, respectively. The $\mathrm{CCD}$ array was cooled to $-40^{\circ} \mathrm{C}$, which greatly reduced dark noise for the exposure durations that we employed. The MTF of the apparatus was measured with an artificial eye consisting of a high-quality lens that imaged the point source on a rotating white diffuser. The rotation of the diffuser served to remove speckle from the aerial image. The apparatus optics were diffraction limited. In addition, the MTF of the CCD camera could be neglected at the higher of the two magnifications. At the lower magnification a small correction was made by use of the CCD MTF provided by Marchywka and Socker. ${ }^{15}$

\section{B. Aligning and Refracting the Eye}

Accommodation was paralyzed as in the interferometric measurements. We used a similar stimulus to align and refract the eye subjectively in both the double-pass and the interferometric measurements. The observer adjusted the horizontal and vertical positions of his bite bar to maximize the contrast of an 18-cycle/deg, horizontal square-wave grating, G. A mirror temporarily placed between the pinhole and lens $L_{1}$ allowed the observer to view the grating, which was sandwiched against a diffuser and was backlit with a $630-\mathrm{nm}$ light. The grating was carefully positioned to lie at the same optical distance from the eye as the pinhole. Lens $\mathrm{L}_{3}$ was attached to the bite bar mount so that the observer could focus the grating by translating his eye together with the lens along the optical axis. This procedure kept the grating magnification constant.

\section{Image Processing}

After an aerial image was acquired, a second image was acquired in exactly the same way but with the eye removed from the system. This second image contained various sources of stray light such as backreflections and scatter from optical elements as well as bias charge on the CCD array. These unwanted effects were removed by subtraction of the second image from the first. Typically, 25 such image pairs would be collected, and the difference images were averaged. We then applied a correction 
(flat fielding) to remove variations in intensity across the field caused by the apparatus and by nonuniformities in the CCD.

We computed the modulus of the Fourier transform of the processed aerial image and then took the square root of the modulus to obtain the single-pass MTF. The single-pass MTF is a two-dimensional (2-D) function, but for the purpose of comparison with the one-dimensional (1-D) interferometric results we used only the slice through the function corresponding to the MTF for horizontal gratings. The point source that we used in our experiments was sufficiently small that no correction of the computed MTF's was made for its finite size. For example, at 50 cycles/deg the loss in contrast attributable to the pinhole was only $\sim 3 \%$.

One assumption underlying the double-pass method is that the second pass through the ocular media is incoherent. Aerial images captured with very brief durations (5 ms) contain laser speckle, indicating a high degree of coherence. ${ }^{1}$ However, the aerial images used in our measurements are recorded over a long (5-s) duration. In this case, eye movements cause the retina to act as a moving diffuser, which greatly reduces coherence.

One concern about the use of long exposures is that the position of the aerial image might change over time owing either to eye movements or to changes in the position of the head on the bite bar. However, control experiments in which the aerial image centroid was computed for a sequence of 5-ms flashes confirm that the position of the aerial image stays quite fixed with respect to the CCD array.

Measurement of the MTF at high spatial frequencies (at which the modulation is small) is limited by spatial noise in the aerial images. We could determine at how high a spatial frequency the MTF is meaningful by computing the phase spectrum of the aerial image. The double-pass technique is constrained to produce an even-symmetric aerial image, so its phase spectrum is zero. ${ }^{16}$ When the signal approaches the noise floor at high frequencies, this flat phase spectrum abruptly becomes erratic. This typically occurred for spatial frequencies higher than $\sim 60$ cycles/deg, so we do not plot our results beyond that value.

\section{DOUBLE-PASS RESULTS}

Figure 4 shows the MTF's obtained with a CCD field of view of $0.8 \mathrm{deg}$ for each of the three observers. We found relatively small differences between MTF's of different observers. The thick solid curve shows the MTF of an optical system with a $3-\mathrm{mm}$ pupil that is limited only by diffraction at $632.8 \mathrm{~nm}$. The thin solid curve shows the results of Campbell and Gubisch ${ }^{4}$ obtained with a $3-\mathrm{mm}$ pupil in white light. At low spatial frequencies, all three MTF's fall more steeply than the data of Campbell and Gubisch. At high frequencies the present estimates are higher than those of Campbell and Gubisch. The reasons for these differences are not clear but may be related to differences among observers, the effect of wavelength on the MTF (see Subsection 7.F below), or chromatic aberration, because Campbell and Gubisch used a broadband source, whereas ours was monochromatic.

\section{DISCUSSION}

\section{A. Comparison of Double-Pass and Interferometric MTF's}

For every observer at every spatial frequency the doublepass modulation transfer was less than that of the interferometric technique. Figure 5 shows the mean interferometric and the mean double-pass MTF's averaged across the three observers. The double-pass MTF drops more steeply than the interferometric MTF between 0 and 20 cycles/deg. Above $\sim 20$ cycles/deg the doublepass MTF stays at roughly $60 \%$ of the interferometric MTF. Given that we used the same observers, pupil size, wavelength, retinal location, and refraction state,

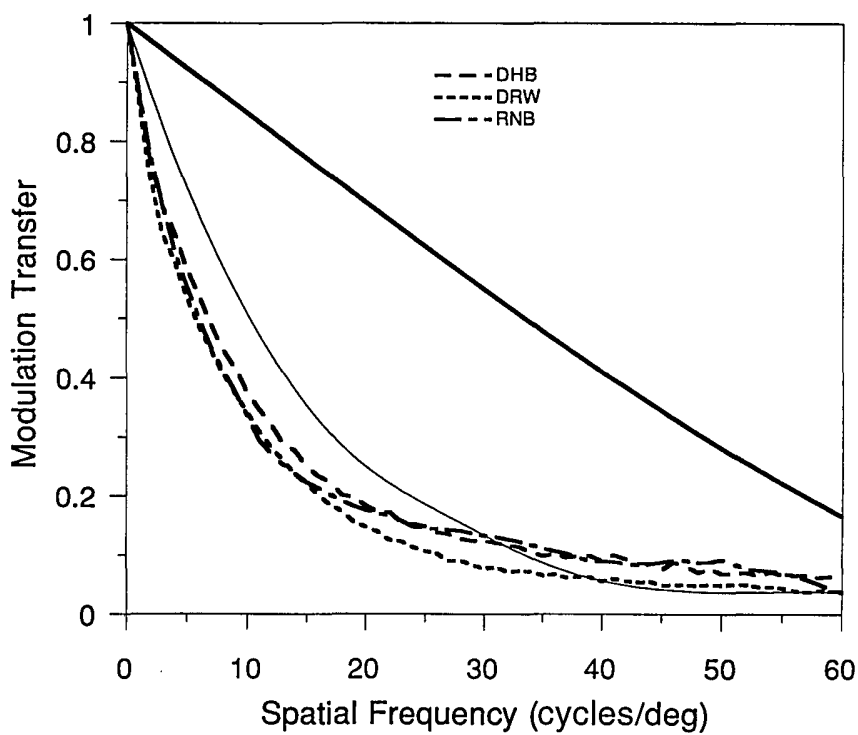

Fig. 4. Double-pass MTF's for three observers, with 632.8-nm wavelength, $3-\mathrm{mm}$ pupil, and 0.8 -deg camera field of view. Heavy solid curve, diffraction-limited MTF for a 3-mm pupil at $632.8 \mathrm{~nm}$; thin solid curve, double-pass MTF of Campbell and Gubisch $^{4}$ obtained with a 3-mm pupil in white light.

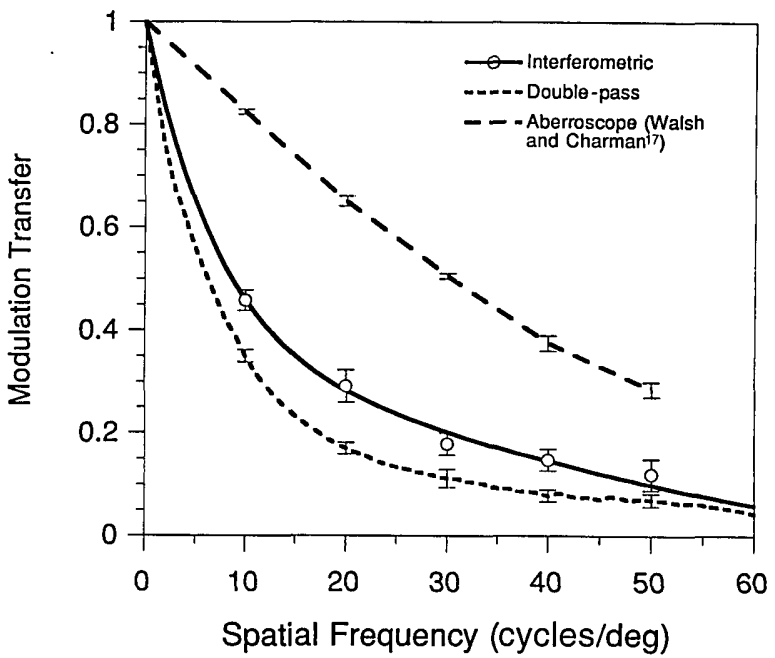

Fig. 5. Comparison of interferometric and double-pass MTF's averaged for the same three observers, refractive state, wavelength, and pupil size. Also shown is the aberroscope MTF, averaged for two observers, of Walsh and Charman. ${ }^{17}$ Error bars show plus and minus one standard error of the mean based on variability among observers. 
none of these factors can explain the difference between the MTF's obtained with the two techniques.

In the double-pass configuration the directional properties of the photoreceptors concentrate the light leaving the pupil. ${ }^{18}$ In principle, this could alter the exit pupil of the eye and influence the double-pass MTF. However, we made measurements of the distribution of light leaving the pupil by imaging it on a CCD array. We found no clear loss in intensity across the pupil, presumably because the pupil was relatively small $(3 \mathrm{~mm})$ and because the Stiles-Crawford function is broader at the foveal center. ${ }^{19}$ Therefore we do not think that the directional sensitivity of the retina plays an important role in the double-pass MTF, at least for this pupil size. We were also concerned that the Stiles-Crawford effect might have influenced our interferometric MTF. As the spatial frequency of an interference fringe is increased, the entry point of the point sources in the pupil becomes more eccentric, which reduces the effective retinal irradiance. In principle this could distort the interferometric contrast sensitivity relative to the incoherent contrast sensitivity, thereby distorting the MTF. In practice this is not a problem, because measurements of the efficiency of interference fringes in one observer were essentially independent of spatial frequency over the frequency range that we used (0-50 cycles/deg). Even a 50-cycles/deg fringe involves a rather small displacement of each point source in the pupil $(0.9 \mathrm{~mm})$.

Figure 6 shows the results in the spatial domain. We calculated point-spread functions (PSF's) corresponding to the interferometric and the double-pass MTF's by fitting the MTF data with the product of the diffraction-limited MTF and the sum of a constant and an exponential [see Eq. (1)]. For the double-pass MTF's the best-fitting parameters were $a=0.1373, w_{1}=0.1998$, and $w_{2}=0.8002$. A 2-D MTF was generated by rotation of the 1-D MTF about the origin. The Fourier transform of this synthesized MTF was then taken as the PSF, assuming cosine phase for all spatial-frequency components. Table 2 provides the PSF calculated from the interferometric data along with the corresponding line-spread function (LSF) for horizontal gratings.

We emphasize that the PSF's should not be interpreted as exact estimates of the actual PSF's. This calculation is only approximate, for two reasons: First, the calculation does not take into account the phase transfer function, which is unknown in our observers. ${ }^{16}$ The effect of assuming cosine phase is to guarantee that the PSF is an even function even if the actual function contains asymmetries that are due to odd aberrations such as coma. Second, the 2-D MTF was generated from the 1-D slice through the MTF, assuming that the former is isotropic. This was done for the interferometric MTF, because no data were obtained at other orientations. For the doublepass data, this procedure provides an estimate of the PSF for which astigmatism has been corrected. Despite these assumptions, the calculation has the virtue that it shows what qualitative differences in the PSF's one anticipates from the differences between the double-pass and the interferometric MTF's.

The PSF's are plotted in Fig. 6 as the fraction of the total light in the point spread per steradian of solid angle. Both the double-pass and the interferometric
PSF's ring slightly as a result of the effect of the diffraction term in the function that is used to fit the data. We do not know whether this slight ringing characterizes the actual PSF. The diffraction-limited PSF is plotted for comparison. The double-pass PSF has relatively more light in the tail than does the interferometric PSF, consistent with its steeper MTF. Both PSF's fall off in a manner that is roughly consistent with the white-light PSF proposed by Vos et al. ${ }^{20}$ which is based in part on psychophysical estimates of glare. These PSF's all show considerably more light in the tails than does the PSF proposed by Westheimer, ${ }^{21}$ suggesting that the latter underestimates scatter at large angles. Westheimer's point-spread estimate may overestimate retinal image contrast even at quite low spatial frequencies.

It has been suggested that the interferometric technique might overestimate the optical quality of the eye. ${ }^{4}$ The assumption underlying this conclusion is that the interferometric technique is insensitive to scattered light because it reduces the contrast of both interference fringes and incoherent gratings. However, scattered light affects interference fringes and incoherent gratings differently. Because interference fringes are formed with coherent light, scatter either from the anterior optics or from the retina forms laser speckle, rather than simple blur, in the retinal image. The appearance of laser speckle in interference fringes is evidence that such scatter must exist. Although the local contrast of an interference fringe is reduced by scatter on average, in some locations it can be decreased and in others it can be increased, depending on the interference of all the light, scattered or otherwise, arriving at each point in the image. To the extent that contrast sensitivity is governed by the regions with highest contrast, the use of interference fringes could conceivably avoid the effect of light scatter. In any case, MacLeod et al..$^{22}$ showed that the MTF of the eye with interference fringes is remark-

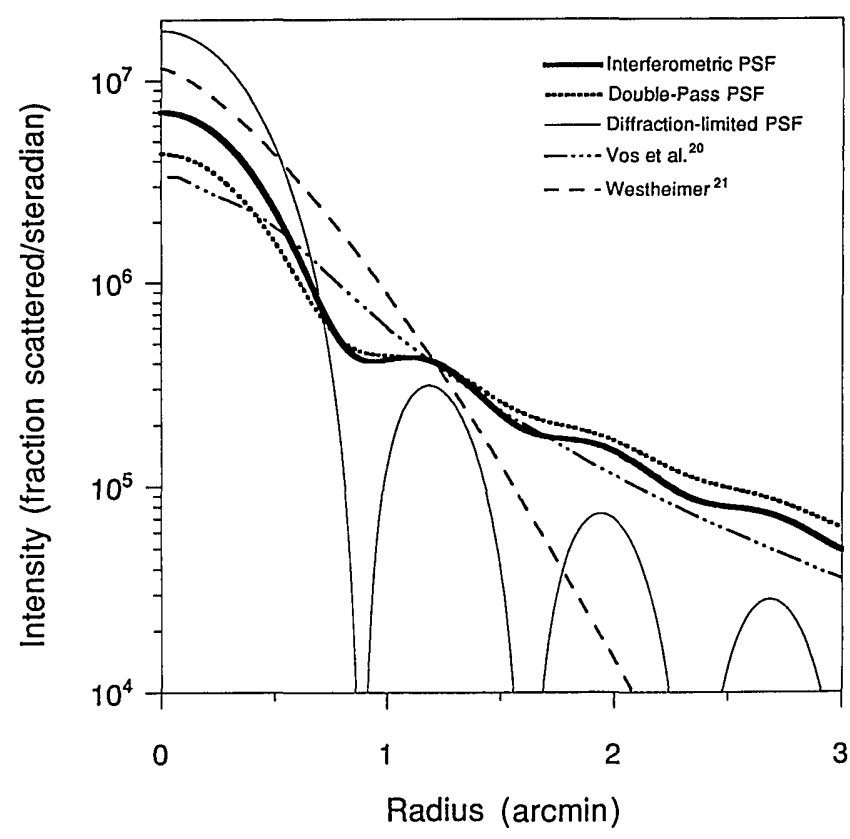

Fig. 6. Comparison of double-pass and interferometric PSF's with the diffraction-limited PSF (Airy disk) and with the PSF's proposed by Vos et al. ${ }^{20}$ and Westheimer. ${ }^{21}$ 
Table 2. Tabulated Values of the PSF and LSF Estimated from the Mean Interferometric MTF ${ }^{a}$

\begin{tabular}{|c|c|c|c|c|c|}
\hline $\begin{array}{l}\text { Distance } \\
\text { (arcmin) }\end{array}$ & PSF & LSF & $\begin{array}{l}\text { Distance } \\
\text { (arcmin) }\end{array}$ & $\mathrm{PSF}\left(\times 10^{4}\right)$ & LSF \\
\hline 0.00 & $6.98 \times 10^{6}$ & $1.95 \times 10^{3}$ & 2.60 & 7.69 & $1.27 \times 10^{2}$ \\
\hline 0.20 & $5.87 \times 10^{6}$ & $1.68 \times 10^{3}$ & 2.80 & 6.47 & $1.11 \times 10^{2}$ \\
\hline 0.30 & $4.71 \times 10^{6}$ & $1.40 \times 10^{3}$ & 2.90 & 5.62 & $1.04 \times 10^{2}$ \\
\hline 0.40 & $3.43 \times 10^{6}$ & $1.10 \times 10^{3}$ & 3.00 & 4.89 & $9.79 \times 10$ \\
\hline 0.50 & $2.27 \times 10^{6}$ & $8.35 \times 10^{2}$ & 3.10 & 4.45 & $9.35 \times 10$ \\
\hline 0.70 & $7.90 \times 10^{5}$ & $5.13 \times 10^{2}$ & 3.30 & 4.17 & $8.63 \times 10$ \\
\hline 0.80 & $5.02 \times 10^{5}$ & $4.52 \times 10^{2}$ & 3.40 & 4.01 & $8.22 \times 10$ \\
\hline 0.90 & $4.12 \times 10^{5}$ & $4.26 \times 10^{2}$ & 3.50 & 3.70 & $7.77 \times 10$ \\
\hline 1.00 & $4.14 \times 10^{5}$ & $4.10 \times 10^{2}$ & 3.60 & 3.29 & $7.33 \times 10$ \\
\hline 1.10 & $4.26 \times 10^{5}$ & $3.89 \times 10^{2}$ & 3.70 & 2.91 & $6.97 \times 10$ \\
\hline 1.20 & $4.06 \times 10^{5}$ & $3.58 \times 10^{2}$ & 3.80 & 2.65 & $6.70 \times 10$ \\
\hline 1.30 & $3.52 \times 10^{5}$ & $3.21 \times 10^{2}$ & 3.90 & 2.54 & $6.48 \times 10$ \\
\hline 1.80 & $1.68 \times 10^{5}$ & $2.16 \times 10^{2}$ & 4.40 & 1.88 & $5.26 \times 10$ \\
\hline 1.90 & $1.61 \times 10^{5}$ & $2.03 \times 10^{2}$ & 4.50 & 1.72 & $5.08 \times 10$ \\
\hline 2.00 & $1.47 \times 10^{5}$ & $1.87 \times 10^{2}$ & 4.60 & 1.64 & $4.94 \times 10$ \\
\hline 2.10 & $1.27 \times 10^{5}$ & $1.71 \times 10^{2}$ & 4.70 & 1.62 & $4.81 \times 10$ \\
\hline 2.20 & $1.06 \times 10^{5}$ & $1.58 \times 10^{2}$ & 4.80 & 1.61 & $4.67 \times 10$ \\
\hline 2.30 & $9.10 \times 10^{4}$ & $1.47 \times 10^{2}$ & 4.90 & 1.54 & $4.50 \times 10$ \\
\hline 2.40 & $8.28 \times 10^{4}$ & $1.40 \times 10^{2}$ & 5.00 & 1.43 & $4.32 \times 10$ \\
\hline 2.50 & $7.93 \times 10^{4}$ & $1.34 \times 10^{2}$ & & & \\
\hline
\end{tabular}

${ }^{a}$ We made two important assumptions to compute these data: (1) that the 2-D MTF is circularly symmetric, although our measurements were restricted to one dimension, and (2) that the PSF is an even function (i.e., that the phase transfer function is zero), although our measurements provide no information about phase. See text for details. To compute the PSF, we used the analytic fit to the measured MTF to generate a circularly symmetric MTF on a $512 \times 512$ pixel grid. We used the fast Fourier transform to compute a raw PSF from the MTF. To generate the table, we extracted a radial slice of the raw PSF and interpolated with a piecewise polynomial. The tabulated PSF is normalized so that it represents the fraction of incident light scattered per steradian. To normalize, we divided the PSF values by the volume under the entire 2-D PSF. The tabulated values may be converted to units of fraction scattered per square degree by multiplication by a factor of $(\pi / 180)^{2}$. To compute the LSF, we used the analytic fit to the MTF to generate a $1-\mathrm{D}$ MTF on a 512-pixel line. We used the fast Fourier transform to compute a raw LSF. To generate the table, we interpolated the raw LSF with a piecewise polynomial. The tabulated LSF is normalized so that it represents the fraction of incident light scattered per radian. To normalize, we divided the LSF values by the area under the entire 1-D LSF. The tabulated values may be converted to units of fraction scattered per degree by multiplication by a factor of ( $\pi / 180$ ).

ably flat, up to very high spatial frequencies, dropping by a factor of 2 at approximately 100 cycles/deg. Even this demodulation is probably largely the result of light integration in foveal cones, leaving little room for much demodulation by light scatter in the eye. Therefore we think that the interferometric MTF captures the important factors that reduce retinal image quality.

We emphasize also that the interferometric MTF is not influenced by neural factors. Our observers could not subjectively distinguish the two types of grating. More important, it is implausible that any part of the visual system beyond the site of photopigment absorption could distinguish them once their contrasts were equated. Thus, when an interference fringe and an incoherent grating of the same spatial frequency are both at contrast threshold, they must have equal contrasts in the retinal image.

The interferometric technique has the advantage that it is based on the light that the eye sees, whereas the double-pass technique relies on light reflected from multiple ocular layers, only one of which is in focus. Thus the expectation is that the double-pass method somewhat underestimates the true MTF, which probably lies closer to the interferometric estimate. The utility of the interferometric estimate is limited by the fact that it is based on measurements on only three observers, and individual differences can be large. ${ }^{23}$ Also, it is one dimensional and it is monochromatic, so that it does not include the effects of chromatic aberration.

\section{B. Comparison with Aberroscope Measurements}

In the aberroscope method ${ }^{17,23-25}$ the image of a rectangular grid in the pupil plane is cast on the retina. Distortions in the grid define the phase errors in the pupil function, from which the MTF can be computed. Figure 5 compares MTF estimates from the objective aberroscope technique ${ }^{17}$ with the present MTF's. The aberroscope MTF is the mean MTF with optimum pupil entry for two observers for horizontal gratings and a 3-mm pupil. Both the double-pass and the interferometric MTF's lie well below the aberroscope estimate. The reasons for this are not clear. The spectral power distribution used in the aberroscope study $(540-660 \mathrm{~nm}$ ) was slightly different, but it seems unlikely that this is of importance. Individual differences may play a role. One computes the aberroscope MTF's by analytically removing the effects of astigmatism and defocus, whereas these aberrations must reduce to some extent the doublepass and the interferometric MTF's, because they are corrected empirically. Another possible reason is that the aberroscope technique might not capture aberrations 
at a fine spatial scale in the pupil plane. There is some indication that image quality may suffer from high-order aberrations in the pupil function, ${ }^{26,27}$ although the contribution of these aberrations is not well established. In any case, light scatter by the anterior optics, which arises partly from refractive-index variations at a microscopic spatial scale, would not be captured by this technique.

A final possibility is that the aberroscope estimate is higher because it does not include retinal scatter. We do not think that retinal scatter is significant, despite measurements of the MTF of excised human retina. ${ }^{28}$ These measurements, which suggest appreciable retinal blurring, were made in post mortem tissue, which clouds rapidly. Furthermore, disarray of photoreceptor orientation in the preparation may also have reduced contrast in these measurements. As mentioned above, in a psychophysical study of the living eye MacLeod et al. ${ }^{22}$ showed that retinal scatter is negligible for interference fringes. In addition, if the inner retina contributed significantly to scatter, one would expect a lower doublepass MTF where the inner retina is thicker. However, Artal and Navarro ${ }^{29}$ found a negligible difference between double-pass MTF's obtained at the foveal center and at 1 -deg eccentricity. We confirmed this result by making measurements at the fovea and several degrees from the fovea, where the retina is thickest. This result provides additional support for the view that retinal scatter is not important.

Methods that capture the wave-front error in the pupil plane, such as the aberroscope method, have important advantages over the interferometric and the double-pass methods. Because phase errors are measured in the pupil plane, the contribution of specific aberrations to image quality can be assessed. Neither the interferometric method nor the double-pass method provides direct information about the aberrations that reduce image quality. Wave-front measurements allow the point-spread function to be calculated for any arbitrary pupil size from a single assessment of the pupil function with use of a large pupil. Furthermore, wave-front methods estimate the phase transfer function, which is an important determinant of image quality. The phase transfer function cannot be determined from either the double-pass ${ }^{16}$ or the interferometric method. Data-collection time is approximately the same with the wave-front method than with the double-pass method and is much quicker than with the interferometric technique. These advantages provide strong incentive to validate a wave-front method to ensure that it captures enough of the eye's aberrations to characterize retinal image quality adequately. To address this issue we are currently making MTF measurements with another wave-front method $^{30}$ that is based on a Hartmann-Shack wave-front sensor.

\section{FACTORS AFFECTING THE DOUBLE-PASS MTF}

The double-pass method has several advantages over the interferometric method. First, it is a far quicker method than the interferometric one. Second, the double-pass method provides the 2-D MTF from a single experimental session, whereas the interferometric technique requires three sessions to estimate the MTF at only five spatial fre- quencies at a single orientation. Third, the double-pass apparatus is easier to construct and operate. Fourth, with the double-pass method it is easier to obtain MTF's outside the fovea, because the poor spatial vision available in the peripheral retina limits the spatial-frequency range of the psychophysical technique. Because of these advantages, we tried various manipulations to identify and remove the additional blur in the double-pass procedure. These are discussed in the remainder of the paper with the hope that they may be of some use to future practitioners of the method. We find that reflections and backscatter from the anterior optics and polarization have relatively small effects on the double-pass MTF, and we discuss methods for handling them. However, the field of view of the CCD camera and the wavelength used have more important effects. With proper choice of experimental conditions the double-pass method can produce MTF's that are quite similar to those of the interferometric method.

\section{A. Comparison of Double-Pass and Subjective Focal Planes}

In the ideal double-pass procedure, one would harvest only those photons that follow the same incoming path as the visually relevant photons absorbed by photopigment. Photons not absorbed would emerge from the apertures of cones, which presumably correspond to the subjective focal plane, with an intensity distribution that faithfully recreated the point spread for incident light. In practice, some fraction of the photons actually harvested are reflected or backscattered from other layers such as the internal limiting membrane or the choroid. If this unwanted fraction were large enough, one would expect that the objective focal plane found in the double-pass procedure might differ from the subjective focal plane.

We compared these focal planes by replacing the CCD camera in the double-pass apparatus with an imageintensified video camera. This device was sufficiently sensitive that the experimenter could focus the aerial image while viewing it in real time on a CRT. ${ }^{31}$ The observer, whose accommodation was paralyzed as before, made similar focus settings while viewing the point source directly. The experimenter and the observer focused with the same micrometer on the bite bar mount. Three observers were tested, with at least four objective and four subjective focus settings made with each. We also made some measurements outside the fovea, where the retina is thicker, to see whether this would shift the objective focus in the direction of the vitreous.

Both the experimenter and the observer found it easy to make focus settings in the fovea. The extrafoveal subjective settings were more difficult because of the reduced resolution there. Table 3 shows the mean dioptric difference between the objective and the subjective focal planes for different observers and retinal locations. A positive number indicates that the objective focal plane was closer to the pigment epithelium than was the subjective focal plane. All the mean differences are close to $0 \mathrm{D}$. In no case was there a significant difference between objective and subjective focal planes. The mean objective focal plane averaged across observers and retinal locations was within $0.01 \mathrm{D}$ from the mean subjective focal plane. If the variability among the six conditions is used to 
Table 3. Mean Objective Minus Mean Subjective Focus in Diopters for Various Observers at Various Retinal Locations

\begin{tabular}{llcc}
\hline $\begin{array}{c}\text { Retinal } \\
\text { Location }\end{array}$ & \multicolumn{1}{c}{ Observer } & $\begin{array}{c}\text { Mean Objective Minus } \\
\text { Mean Subjective Focus } \\
\text { (D) }\end{array}$ & $\begin{array}{c}\text { Standard Error of the } \\
\text { Mean Difference }\end{array}$ \\
\hline Fovea & DRW & -0.01 & 0.08 \\
& RNB & 0.00 & 0.17 \\
EA & DRW 4-deg nasal & 0.07 & 0.04 \\
& DRW 8-deg inferior & 0.08 & 0.09 \\
& RBN 4.4-deg nasal & -0.14 & 0.10 \\
& Mean & -0.05 & 0.33 \\
& Standard error & -0.01 & 0.03 \\
\hline
\end{tabular}

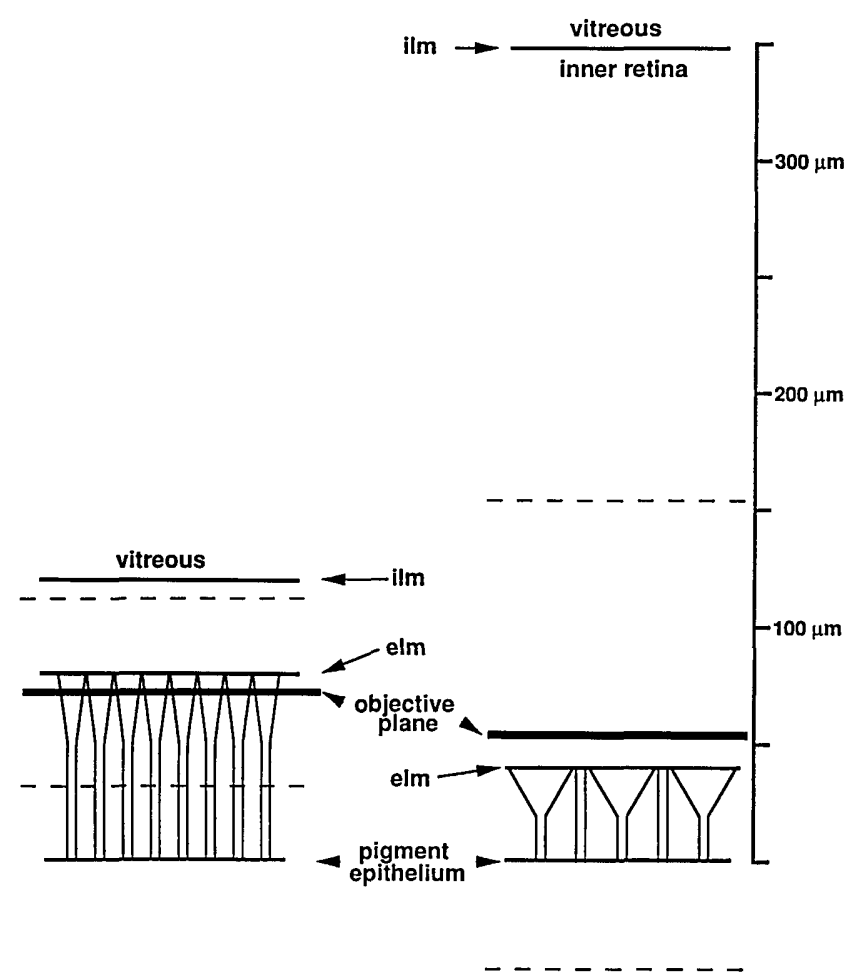

FOVEA

Fig. 7. Comparison of subjective and objective focal planes obtained with the double-pass method. The subjective focal plane is assumed to lie at the external limiting membrane, elm. Dashed lines show the 95\% confidence interval bracketing the mean objective plane, shown as heavy horizontal lines; ilm, inner limiting membrane.

estimate the sensitivity of the technique, the smallest discrepancy that we would have been able to detect reliably is $\sim 0.08 \mathrm{D}$ ( $\alpha=0.05$, two-tailed t-test, five degrees of freedom). If we assume that $1 \mathrm{D}$ corresponds to a $371-\mu \mathrm{m}$ axial shift in the retinal image, this would correspond to $\sim 30 \mu \mathrm{m}$.

Figure 7 shows the results separately for foveal and extrafoveal locations expressed in retinal distances instead of diopters. We assume that the subjective focal plane corresponds to the external limiting membrane. ${ }^{32}$ Thick horizontal lines indicate the objective focal plane, and the dashed lines define the $95 \%$ confidence interval.
We assume a foveal cone length of $\sim 80 \mu \mathrm{m}$ (Ref. 33 ) and an extrafoveal receptor length of half that value. Retinal thickness estimates were taken from Fig. 161 of Ref. 34 .

The data for the fovea reject the hypothesis that the mean objective focal plane lies at the internal limiting membrane or in the pigment epithelium. For the extrafovea the confidence interval is wider, but the internal limiting membrane can still be rejected as the objective focal plane. The light returning from the retina is directional, ${ }^{18,35-37}$ implying that a substantial fraction is waveguided within receptors. This waveguiding must largely preserve the distribution of light in the point source at the entrance apertures of the cones, causing the subjective and objective focal planes to agree. Apparently the sources of additional blurring in the doublepass method are subtle enough that they do not produce a shift in the objective focal plane away from the plane of subjective focus.

Glickstein and Millodot ${ }^{38}$ suggested that in retinoscopy most of the light reflected from the retina arises at the inner limiting membrane. They invoked this hypothesis to explain a difference between retinoscopic and subjective measures of refractive state. Our results do not support this view, agreeing with the conclusions of Charman. ${ }^{39}$

\section{B. Effect of the Purkinje Images}

A potential source of extraneous light in the double-pass technique comes from light reflected back from the various surfaces of the anterior optics, i.e., the light that forms the Purkinje images. The reflection from the first surface of the cornea would make the predominant contribution to this contaminating source of light, because it far outweighs the reflections from the other surfaces. The virtual image of the point source formed by the cornea is out of focus with respect to the CCD array and therefore produces a uniform background in the aerial image. This increases the total amount of light collected, so that the computed MTF has a precipitous drop at the lowest spatial frequencies. The larger the field of view of the CCD, the larger the drop. When the MTF is normalized to unity at zero spatial frequency, the uniform veil of light caused by the Purkinje images reduces the apparent modulation at other spatial frequencies. An abrupt drop in the MTF at the first nonzero spatial frequencies is a signature of the Purkinje images in the MTF. This signature does not appear in the double-pass MTF's of Fig. 4, 
so the Purkinje images cannot be invoked to explain the discrepancy between the interferometric and the doublepass MTF's. Direct observations of the location of the corneal reflex relative to the artificial pupil for two of the observers (DHB and DRW) showed that the alignment of the eye that produced the optimum image quality caused the artificial pupil to block the corneal reflex. We suspect that this must have been true for the third observer, as well. For some observers the entry point for optimum image quality is displaced at least $1.5 \mathrm{~mm}$ from the corneal pole.

In cases in which the corneal reflex is not blocked, it is possible to measure and subtract this unwanted signal. An aperture was placed in the focal plane between lenses $L_{2}$ and $L_{3}$ (Fig. 3) that was conjugate with the CCD. The aperture was registered with and had the same magnification as the CCD, so the only light that could pass through the system was that which would have fallen on the CCD. We then replaced lens $\mathrm{L}_{4}$ with another lens of half the focal length so the artificial pupil was imaged on the CCD instead of the retina. In this way we could collect images of the light distribution in the pupil under conditions in which the corneal and retinal contributions were identical to those when aerial images were collected. In the pupil plane the corneal reflex appears as a bright point amid the diffuse glow of light returning largely from the retina. With the camera field of view at $1.6 \mathrm{deg}$ and $632.8 \mathrm{~nm}$, the corneal reflex accounted for approximately one quarter to one third of the light in the aerial image, depending on the observer. At $543 \mathrm{~nm}$ the decreased reflectance of the fundus increased the corneal fraction to approximately half.

\section{Effect of Backscatter by the Anterior Optics}

In addition to creating specular reflections, the anterior optics might also have degraded the aerial image by backscatter. We estimated the backscatter in two observers by illuminating only one half of the pupil and measuring the difference in the intensity of the light emerging from the two halves of the pupil on the return pass. The retinal component fills the entire pupil, but the backscatter appears only in the illuminated half. Therefore the difference in the amount of light returning from the illuminated and the unilluminated halves of the pupil provides an estimate of the backscattered light. Of all the light returning through the pupil, backscatter by the anterior optics could account for only $7 \%$ for observer DRW and $3 \%$ for observer DHB. This would produce a drop in the MTF at very low spatial frequencies of less than $4 \%$ and $2 \%$, respectively, too small to account for the difference between the interferometric and the double-pass results. Because light reflected and scattered back from the anterior optics plays a negligible role in the doublepass MTF measurements presented in Figs. 4 and 5, the additional image degradation found in the double-pass MTF must be caused largely by the fundus.

\section{Effect of Field Size}

Simon and Denieul ${ }^{40}$ showed that the failure to collect the entire skirt in the aerial image can lead to an overestimation of the double-pass MTF. Truncation of the edges of the aerial image causes an underestimate of the total amount of light in the image, which is the square root of the modulus of the Fourier transform at zero spatial frequency. The MTF is normalized to unity at zero spatial frequency, causing an artificially high estimate of the modulation transfer at nonzero spatial frequencies. This effect is illustrated in Fig. 8, which shows MTF's computed from an aerial image acquired with a 1.6-deg instead of a 0.8-deg field of view. We doubled the field size by halving the focal length of lens $\mathrm{L}_{4}$. We then manipulated the field size further by truncating the average aerial image by various amounts before computing the MTF. The modulation transfer decreases at all frequencies as the field of view is increased from 0.2 to $1.6 \mathrm{deg}$. Even the 1.6-deg field of view, the largest that we tried, did not capture all of the aerial image skirt. The intensity remained slightly above zero at the very edges of the image, suggesting that we slightly overestimated the MTF that can actually be obtained with the double-pass technique at this wavelength. All double-pass MTF's are subject to this problem. It emphasizes the need for a detector with a large enough dynamic range to capture the absolute irradiance in the tails of the aerial image, even when it is several orders of magnitude below the irradiance at the peak.

\section{E. Effect of Polarization}

The double-pass MTF's plotted in Fig. 4 were based on measurements in which the incident beam was vertically polarized but the return path contained no polarizer. Rohler et $a l . .^{5}$ reported that the double-pass modulation transfer was greater when the light reflected from the fundus was polarized parallel rather than perpendicular to the incident beam. We therefore made some additional observations to determine whether the addition of a polarizer in the return path could remove the discrepancy between the double-pass and the interferometric MTF's. We confirmed the effect that Rohler et al. described, by

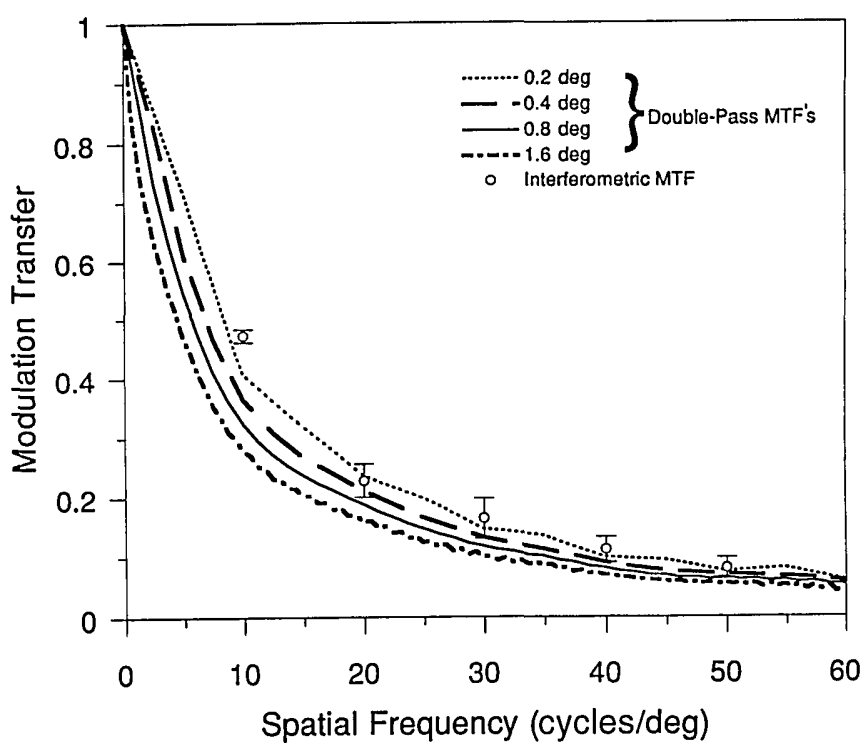

Fig. 8. Double-pass MTF's obtained with observer DRW in 632.8-nm light, showing that reducing the CCD camera field of view spuriously increases the MTF. The linear polarizer that was placed in the output path was oriented parallel to the polarization axis of the input beam. Shown for comparison is the interferometric MTF for observer DRW. 
measuring MTF's with either a crossed or an uncrossed polarizer between lens $\mathrm{L}_{4}$ and the CCD camera. However, because most of the reflected light retains its polarization, ${ }^{41,42}$ the depolarized component has little effect on the MTF's of Fig. 4. Measurements made on two observers showed that the addition of a parallel linear polarizer in the return path elevated the MTF by a constant amount at all spatial frequencies but was small, never more than $10 \%$. A polarizer is recommended for doublepass measurements because it does reduce the unwanted skirt in the aerial image. However, the data shown in Fig. 8 were collected with use of such a polarizer, showing that it is insufficient to bring the double-pass MTF into correspondence with the interferometric results. We did not perform any experiments in which the birefringent effects of the optic media were compensated. ${ }^{43}$

\section{F. Effect of Wavelength}

The reddish hue of the fundus image ensures that some of the light in the double-pass method arises from behind the receptors. One would therefore expect a decline in the double-pass MTF as the light penetrates deeper into the choroid. Because green light does not penetrate the choroid so effectively as red light and is scattered less by the fundus, one would suspect that its use might increase double-pass modulation transfer. Westheimer and Campbell ${ }^{44}$ observed that the aerial image had a broader tail in long-wavelength light. Furthermore, Charman and Jennings ${ }^{45}$ found that the doublepass MTF declined most slowly with spatial frequency in the yellow-to-green part of the spectrum, dropping more precipitously for both long- and short-wavelength light. Figure 9 shows the MTF's for observer DRW obtained with 543-nm light at four field sizes and with $632.8-\mathrm{nm}$ light obtained with a 1.6-deg field. The MTF at $543 \mathrm{~nm}$ is clearly higher than that at $632.8 \mathrm{~nm}$. Shown also is the effect of truncation of the aerial image at $543 \mathrm{~nm}$. Although there is some effect, it is substantially smaller than that obtained with 632.8-nm light (see Fig. 8), confirming earlier reports that the aerial image is somewhat more compact in green light. Similar results were obtained for a second observer.

The comparison is complicated somewhat by the fact that diffraction blurs the 632.8-nm MTF more than it does the 543-nm MTF, and it is not possible to correct exactly for the effect of diffraction without knowledge of the wave aberrations in the pupil plane. However, we can correct the MTF approximately by multiplying the 543-nm MTF by the ratio of the 632.8- to the 543-nm diffraction-limited MTF's at this pupil size. Figure 10 shows both the original 543-nm MTF and one with the approximate correction for diffraction. The double-pass MTF for this observer in 543-nm-wavelength light agrees well with the interferometric MTF obtained at longer wavelengths. We have not made any measurements in 543-nm light with the interferometric method to determine whether the MTF is similarly raised.

We were also concerned that this effect of wavelength was related to photopigment absorption, which is higher in the middle of the spectrum. Specifically, we hypothesized that pigment bleaching in retinal locations underlying the core of the point source could increase the irradiance of the aerial image core relative to its skirt.
This bleaching, however, did not artificially increase the MTF in green light; additional experiments showed that bleaching all the photopigment before collecting each aerial image had no effect on the double-pass MTF.

The fact that structures associated with both the inner retina and the choroid can be discerned in fundus photographs ensures that light in the double-pass procedure is reflected from layers both in front of and behind the receptors. However, the relative contributions of these reflections to the aerial image are not yet well quantified. As we mentioned earlier, Artal and Navarro ${ }^{29}$ found that the double-pass MTF is little different at the foveal

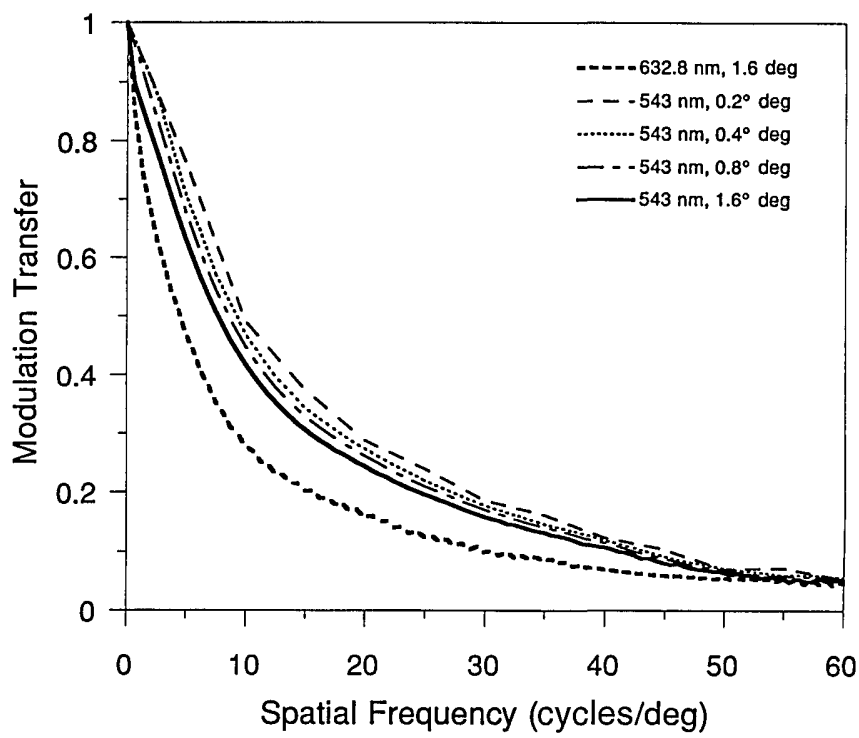

Fig. 9. Comparison of double-pass MTF's obtained at 632.8- and 543-nm wavelengths for observer DRW. Also shown is the effect of CCD field size for the 543-nm case; this effect is smaller than the effect at $632.8 \mathrm{~nm}$ shown in Fig. 8.

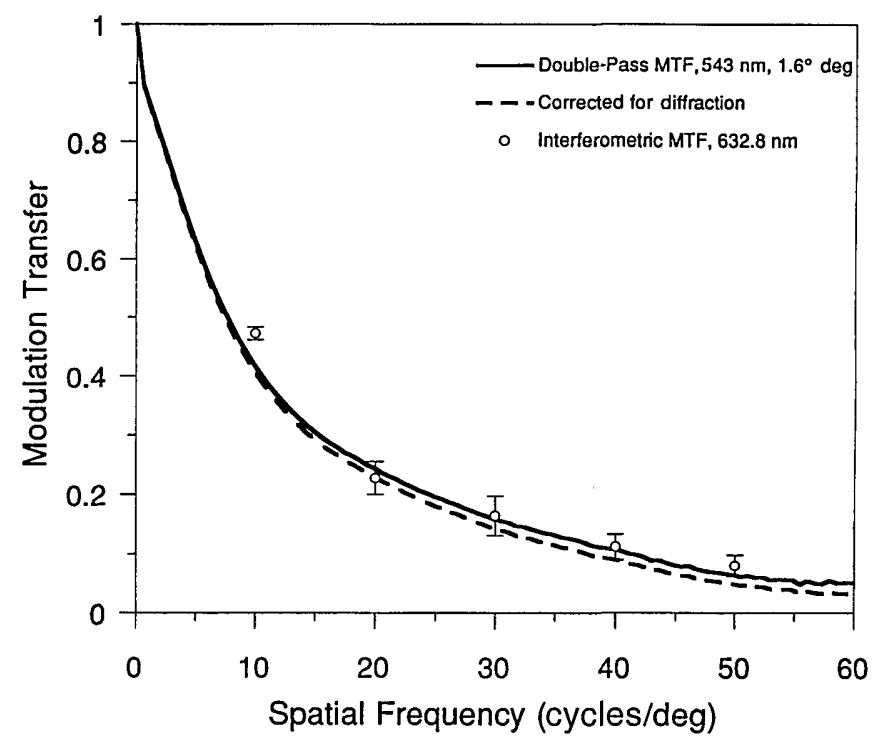

Fig. 10. Comparison of the double-pass MTF obtained with 543-nm light and the interferometric MTF at $632.8 \mathrm{~nm}$ for observer DRW. The dashed curve shows the approximate double-pass MTF that would have been expected if the blurring by diffraction had been at 632.8 instead of $543 \mathrm{~nm}$. 
center and just outside it, where the inner retina is substantially thicker, a result that we have confirmed with additional experiments. These results suggest that a relatively small portion of the light comes from the inner retina. In red light and with normal young eyes, for which scattering by the anterior optics is not so great, we suggest that most of the additional image degradation in the double-pass procedure is likely caused by fundal scattering, probably in the choroid. It is possible that much of the light scattered in this way is not visually effective. The light scattered back from the choroid probably does not couple efficiently into the cone photoreceptors because, unlike incoming light, it does not have access to the light-funneling properties of the cone inner segments.

\section{ACKNOWLEDGMENTS}

Much of the earlier work on this project was completed at and with the material support of the Instituto de Optica, Madrid, Spain, where CCD arrays were first used in the double-pass procedure. We are grateful to Pablo Artal, Melanie Campbell, Neil Charman, and Brian Wandell for helpful discussions and to Nobu Sekiguchi for constructing the interferometer used in this work. The research was also supported by National Institutes of Health grants EY01319 and EY04367, National Research Service Award fellowship EY06278 to D. Brainard, and Comisión Interministerial de Ciencia y Technología (Spain) grant TIC91-0438 to R. Navarro.

\section{REFERENCES}

1. J. Santamaria, P. Artal, and J. Bescós, "Determination of the point-spread function of human eyes using a hybrid optical-digital method," J. Opt. Soc. Am. A 4, 1109-1114 (1987).

2. F. Flamant, "Etude de la repartition de lumiére dans l'image rétinienne d'une fente," Rev. Opt. Theor. Instrum. 34, 433-459 (1955).

3. J. Krauskopf, "Light distribution in human retinal images," J. Opt. Soc. Am. 52, 1046-1050 (1962).

4. F. W. Campbell and R. W. Gubisch, "Optical quality of the human eye," J. Physiol. (London) 186, 558-578 (1966).

5. R. Rohler, U. Miller, and M. Aberl, "Zur Messung der Modulationsubertragungsfunktion des lebenden menschlichen Auges im Reflektierten Licht," Vision Res. 9, 407-428 (1969).

6. R. Navarro, P. Artal, and D. R. Williams, "Modulation transfer of the human eye as a function of retinal eccentricity," J. Opt. Soc. Am. A 10, 201-212 (1993).

7. F. W. Campbell and D. G. Green, "Optical and retinal factors affecting visual resolution," J. Physiol. (London) 181, 576-593 (1965).

8. A. Arnulf and O. Dupuy, "La transmission des contrastes par le système optique de l'oeil et les seuils des contrastes rétiniens," C. R. Acad. Sci. (Paris) 250, 2757-2759 (1960).

9. S. Berger-Lheureux-Robardey, "Mesure de la fonction de transfert de modulation du système optique de l'oeil et des seuils de modulation rétiniens," Rev. Opt. Theor. Instrum. 44, 294-323 (1965).

10. N. Sekiguchi, D. R. Williams, and D. H. Brainard, "Aberration-free measurements of the visibility of isoluminant gratings," J. Opt. Soc. Am. A 10, 2105-2117 (1993).

11. D. R. Williams, "Visibility of interference fringes near the resolution limit," J. Opt. Soc. Am. A 2, 1087-1093 (1985).

12. A. B. Watson and D. G. Pelli, "QUEST: a Bayesian adaptive psychometric method," Percept. Psychophys. 33, 113-120 (1983).

13. J. W. Goodman, Introduction to Fourier Optics (McGrawHill, San Francisco, Calif., 1968).

14. D. H. Sliney and M. L. Wolbarsht, "Safety standards and measurement techniques for high intensity light sources," Vision Res. 20, 1133-1142 (1980).

15. M. Marchywka and D. G. Socker, "Modulation transfer function measurement technique for small-pixel detectors," Appl. Opt. 31, 7198-7213 (1992)

16. P. Artal, S. Marcos, R. Navarro, and D. R. Williams, "Odd aberrations and double-pass measurements of retinal image quality," J. Opt. Soc. Am. A (to be published).

17. G. Walsh and W. N. Charman, "The effect of pupil centration and diameter on ocular performance," Vision Res. 28, 659-665 (1988).

18. G. J. van Blokland, "Directionality and alignment of the foveal receptors, assessed with light scattered from the human fundus in vivo," Vision Res. 26, 495-500 (1986).

19. G. Westheimer, "Dependence of the magnitude of the Stiles-Crawford effect on retinal location," J. Physiol. 192, 309-315 (1967).

20. J. J. Vos, J. Walraven, and A. Van Meeteren, "Light profiles of the foveal image of a point source," Vision Res. 16, 215-219 (1976).

21. G. Westheimer, "The eye as an optical instrument," in Handbook of Perception and Human Performance, K. R. Boff, L. Kaufman, and J. P. Thomas, eds. (Wiley, New York, 1986), Vol. 1 , pp. $4 / 1-4 / 20$.

22. D. I. A. MacLeod, D. R. Williams, and W. Makous, "A visual nonlinearity fed by single cones," Vision Res. 32, 347-363 (1992).

23. G. Walsh and W. N. Charman, "Objective technique for the determination of monochromatic aberrations of the human eye," J. Opt. Soc. Am. A 1, 987-992 (1984).

24. H. C. Howland and B. Howland, "A subjective method for the measurement of monochromatic aberrations of the eye," J. Opt. Soc. Am. 67, 1508-1518 (1977).

25. G. Walsh and W. N. Charman, "Measurement of the axial wavefront aberration of the human eye," Ophthal. Physiol. Opt. 5, 23-31 (1985).

26. F. Berny and S. Slansky, "Wavefront determination resulting from Foucault test as applied to the human eye and visual instruments," in Optical Instruments and Techniques, J. H. Dickenson, ed. (Oriel, Newcastle, UK, 1969), pp. $375-386$.

27. H. C. Howland and J. Buettner, "Computing high order wave aberration coefficients from variations of best focus for small artificial pupils," Vision Res. 29, 979-983 (1989).

28. H. Ohzu and J. M. Enoch, "Optical modulation by the isolated human fovea," Vision Res. 12, 245-251 (1972).

29. P. Artal and R. Navarro, "Simultaneous measurement of two point-spread functions at different locations across the human fovea," Appl. Opt. 31, 3646-3656 (1992).

30. J. Liang, B. Grimm, S. Goelz, and J. Bille, "Objective measurement of wave aberrations of the human eye with use of a Hartmann-Shack wave-front sensor," J. Opt. Soc. Am. A 11, 1949-1957 (1994).

31. A. Arnulf, J. Santamaria, and J. Bescós, "A cinematographic method for the dynamic study of the image formation by the human eye. Microfluctuations of the accommodation," J. Opt. (Paris) 12, 123-128 (1981).

32. B. Chen, W. Makous, and D. R. Williams, "Serial spatial filters in vision," Vision Res. 33, 413-427 (1993).

33. C. Yuodelis and A. Hendrickson, "A qualitative and quantitative analysis of the human fovea during development," Vision Res. 26, 847-876 (1986).

34. S. Polyak, The Vertebrate Visual System (U. Chicago Press, Chicago, Ill., 1957).

35. H. Goldmann, "Stiles-Crawford effekt," Ophthalmologica 103, 225-229 (1942).

36. J. Krauskopf, "Some experiments with a photoelectric ophthalmoscope," in Performance of the Eye at Low Luminances, M. A. Bouman and J. J. Vos, eds. Excerpta Medica International Congress Series, No. 125 (Excerpta Medica Foundation, Amsterdam, 1966). 
37. J.-M. Gorrand and F. C. Delori, "Reflectometric technique for assessing photoreceptor alignment," Vision Res. (to be published).

38. M. Glickstein and M. Millodot, "Retinoscopy and eye size," Science 168, 605-606 (1970).

39. W. N. Charman, "Some sources of discrepancy between static retinoscopy and subjective refraction," Brit. J. Physiol. Opt. 30, 108-118 (1975).

40. J. F. Simon and P. M. Denieul, "Influence of the size of test field employed in measurements of modulation transfer function of the eye," J. Opt. Soc. Am. 63, 894-896 (1973).

41. R. A. Weale, "Polarized light and the human fundus oculi," J. Physiol. 186, 175-186 (1966).
42. G. J. van Blokland, "Ellipsometry of the human retina in vivo: preservation of polarization," J. Opt. Soc. Am. A 2, $72-75$ (1985).

43. L. J. Bour, "Polarized light and the eye," in Vision and Visual Dysfunction, J. R. Cronly-Dillon, ed. (Macmillan, New York, 1991), Vol. 1, pp. 310-325.

44. G. Westheimer and F. W. Campbell, "Light distribution in the image formed by the living human eye," J. Opt. Soc. Am. 52, 1040-1044 (1962).

45. W. N. Charman and J. A. M. Jennings, "The optical quality of the retinal image as a function of focus," Br. J. Physiol. Opt. 31, 119-134 (1976). 